James J. Cox, C. Geoffrey Woods, and Ingo Kurth*

\title{
Peripheral sensory neuropathies - pain loss vs. pain gain
}

https://doi.org/10.1515/medgen-2020-2039

Received May 11, 2020; accepted September 3, 2020

\begin{abstract}
Peripheral sensory neurons are afferent neurons that innervate the skin, joints, bones, muscles, and mucosal tissues. By converting different stimuli into action potentials, they transmit signals for the sensing of temperature, touch, pressure, or pain. This review discusses the known Mendelian disorders which affect pain sensing in humans. For painlessness, these disorders can be classified as developmental, neurodegenerative, or functional, where pain-sensing neurons (nociceptors) are present but cannot be activated or produce action potentials. Affected patients suffer from numbness with recurrent injuries, burns, and poorly healing wounds. For Mendelian disorders of excess pain, aberrant overactivity of nociceptors is a hallmark and leads to paroxysmal or continuous pain states. Again, the effect can be functional or, as in small fiber neuropathies, can be accompanied by degeneration of small unmyelinated nerve fibers in the skin. About 20 different genes are known to cause Mendelian pain disorders and the molecules involved are of general interest for human pain research and as analgesic targets. Comprehensive genetic testing is the key to early diagnosis and adaptation of clinical management.
\end{abstract}

Keywords: hereditary sensory and autonomic neuropathy, congenital insensitivity to pain, small fiber neuropathy, sodium channels

\section{Introduction}

Peripheral sensory nerves transmit stimuli from the environment. Different subtypes of cutaneous afferent nerves mediate temperature, touch, pressure, vibration, or pain. The latter is mediated by small A $\delta$-fibers and unmyeli-

\footnotetext{
*Corresponding author: Ingo Kurth, Institute of Human Genetics, Medical Faculty, Uniklinik RWTH Aachen, Pauwelsstr. 30, 52074 Aachen, Germany, e-mail: ikurth@ukaachen.de

James J. Cox, Molecular Nociception Group, Wolfson Institute for Biomedical Research, University College London, London, UK, e-mail: j.j.cox@ucl.ac.uk

C. Geoffrey Woods, Cambridge Institute for Medical Research, The Clinical Medical School, University of Cambridge, Cambridge, UK, e-mail:cw347@cam.ac.uk
}

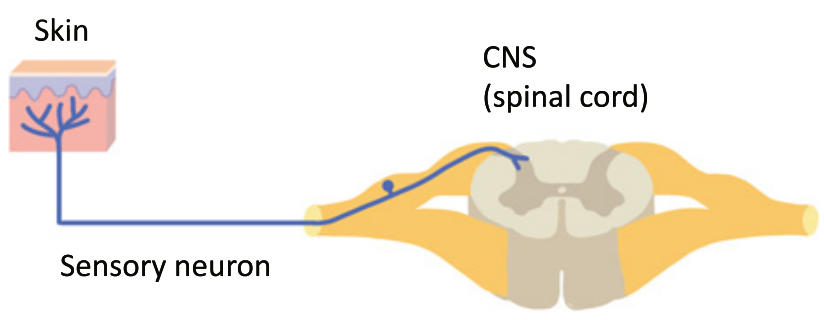

Figure 1: Sensory neurons are afferent nerves and stimulation at the distal end (skin) leads to action potential generation. Higherorder neurons in the dorsal horn of the central nervous system (CNS) transmit the signal to the brain. The cell bodies of these pseudounipolar neurons are bundled in dorsal root ganglia (DRGs) along the vertebral column.

nated C-fibers (nociceptors). Sensory neurons are highly specialized since they bridge a distance of up to one meter between the skin and their central projections in the spinal cord (Figure 1). They are equipped with a large repertoire of transport proteins, neurotrophin receptors, and ion channels that are involved in the development, long-term survival, and excitability of sensory neurons. Dysfunction or neurodegeneration of sensory neurons can result in opposite clinical phenotypes ranging from pain insensitivity to severe episodic pain or painful chronic neuropathies. Respective Mendelian disorders with pain loss are classified as congenital insensitivity to pain (CIP) or hereditary sensory and autonomic neuropathy (HSAN), whereas disorders with increased pain include primary erythromelalgia, familial episodic pain syndromes, and small fiber neuropathies (SFN). In this review on human Mendelian nociception we will discuss clinical symptoms, genes, pathomechanisms, and how these data have been used for novel analgesic development.

\section{Pain loss}

\section{Congenital insensitivity to pain (CIP)}

Mendelian human pain insensitivity disorders are reported whereby patients present with a normal intraepidermal nerve fiber density, but recently lack of C-fiber nociceptors has been reported in some patients [1]. These disorders, CIP, are caused by mutations in voltage-gated sodium 


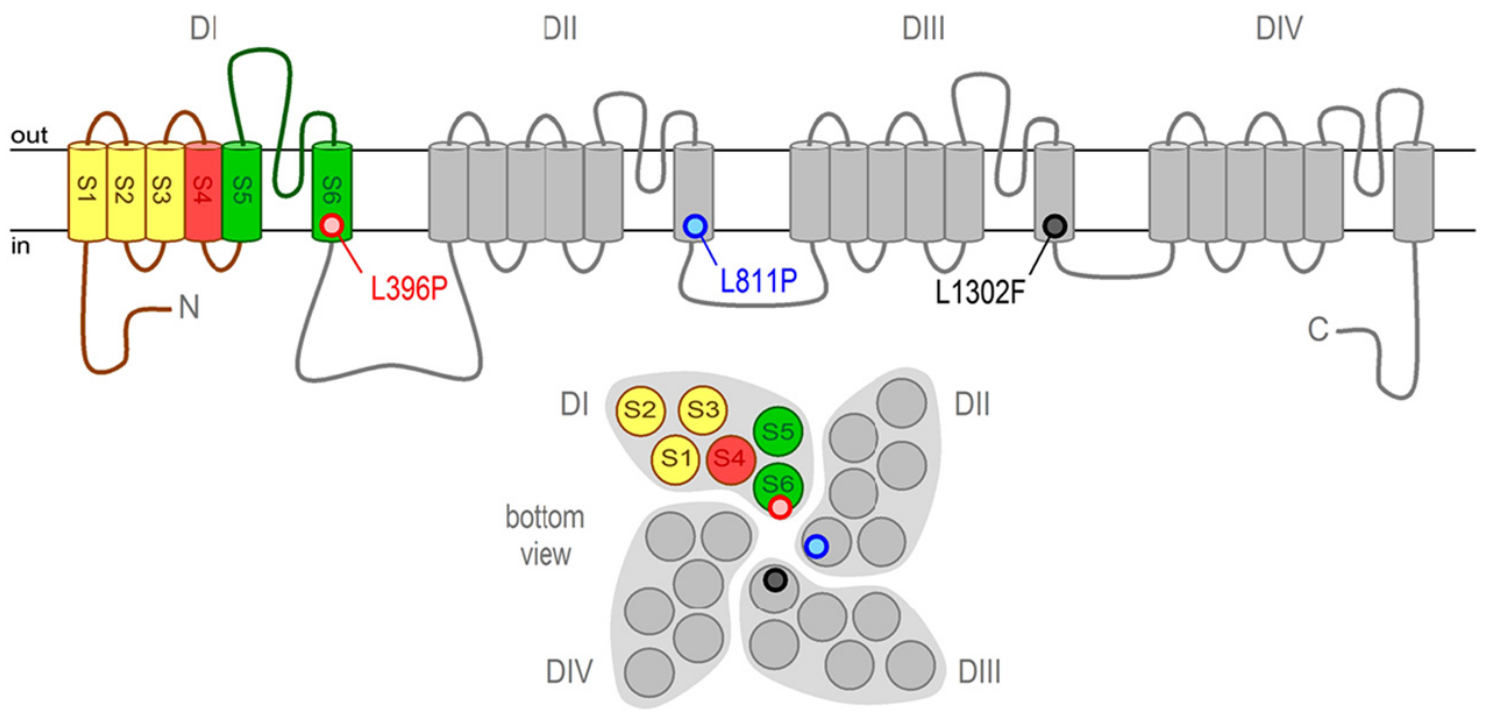

Figure 2: Figure from [7]: Tight balance between pain gain and loss in Nav1.9 disorders: Mutations in the distal S6 segments of the channel's DI-DIV domains are linked to human pain insensitivity (p.Leu396Pro, p.Leu811Pro, p.Leu1302Phe), whereas mutations of other amino acids cause episodic pain, cold-induced pain, or small fiber neuropathy.

channel genes SCN9A or SCN11A, the zinc finger homeobox 2 gene (ZFHX2), or the pseudogene FAAH-OUT (Table 1). There is considerable clinical overlap with HSAN disorders which are discussed below.

Individuals with bi-allelic loss-of-function mutations in SCN9A, encoding the voltage-gated sodium channel $\mathrm{Na}_{\mathrm{V}} 1.7$, are born with a complete inability to experience pain and are also anosmic (lack a sense of smell) [2-4]. Painlessness leads to biting of the lips, tongue, and fingers. Cuts and painless bone fractures are common. Touch, warm and cold temperatures, proprioception, tickle, and pressure are correctly perceived and patients have normal intelligence. Visceral pains are not perceived, including painless labor. Sensory nerves typically show normal structure [2], but a subgroup of patients shows morphological abnormalities of sensory nerves and were classified as having HSAN Type IID [5]. Na 1.7 amplifies slowly developing subthreshold depolarizing inputs and acts as generator for action potentials in nociceptors.

A crucial role for SCN11A in human pain perception was first demonstrated by a mutation that causes heritable painlessness [6]. Patients with SCN11A-related pain insensitivity have normal intelligence, but several additional symptoms, such as diarrhea or severe constipation, muscle hypotonia, extreme itch, and increased sweating. Like all voltage-gated sodium channels, $\mathrm{Na}_{\mathrm{V}} 1.9$, encoded by SCN11A, is composed of four homologous domains (DI-DIV), each organized by six transmembrane segments (S1-S6) (Figure 2). $\mathrm{Na}_{\mathrm{V}} 1.9$ has ultraslow kinetics and acts as a threshold channel that contributes to the resting po- tential of neurons. Interestingly, the phenotype of $\mathrm{Na}_{\mathrm{V}} 1.9$ pain-insensitivity is caused only by specific missense mutations (Figure 2) [6-8]. These mutations lead to dramatic gain-of-function characteristics on a channel level and increase the resting membrane potential of neurons which subsequently may silence the nociceptors. Interestingly, other gain-of-function mutations in $\mathrm{Na}_{V} 1.9$ are reported to lead to episodic pain and small fiber neuropathies [9-11]. The opposite clinical outcome may depend on the degree of membrane depolarization: Larger membrane depolarizations result in hypoexcitability and pain insensitivity, and smaller depolarizations result in hyperexcitability and pain (see also the section on "Pain Gain").

Hyposensitivity to painful thermal and capsaicin stimulation and painless injuries from childhood have been reported in an autosomal-dominant disorder (Marsili syndrome) and a mutation in ZFHX2, a transcriptional regulator expressed within damage-sensing primary afferents, was identified [12]. Gene expression analyses in dorsal root ganglia from mutant and wild-type mice showed altered expression of genes implicated in peripheral pain mechanisms. A punch skin biopsy in a patient showed a normal intraepidermal nerve fiber density, indicating that the phenotype is not due to a neuropathy.

Another interesting pain loss mechanism is a microdeletion in the pseudogene and long non-coding RNA FAAH-OUT, which results in pain insensitivity and impaired anxiety [13]. This non-coding RNA regulates the neighboring $F A A H$ gene, which is involved in endocannabinoid signaling [14]. The genetic findings and ele- 


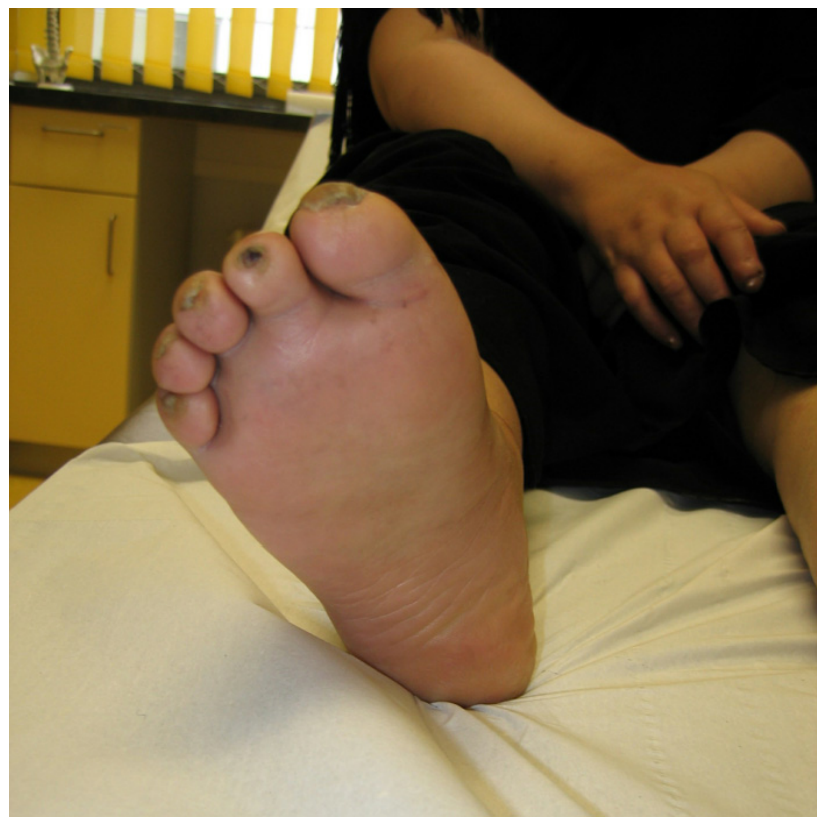

Figure 3: Patient with HSAN and tissue damage.

vated circulating fatty-acid amides in the peripheral blood of the patient is consistent with a phenotype resulting from enhanced endocannabinoid signaling and a loss of function of $F A A H$.

\section{Hereditary sensory and autonomic neuropathies (HSAN)}

The different subtypes of HSAN are characterized by neurodegeneration and/or neurodevelopmental defects of sensory neurons. Subtypes of HSAN1 follow an autosomal dominant inheritance, whereas HSAN2-8 are inherited autosomal recessively [15]. Dominant forms usually manifest later in life than recessive forms, which are often congenital. The clinical symptoms of the recessive forms are usually more diverse and are characterized by severe complications. Children with congenital lack of pain sensation tend to behave in a self-injuring manner, as avoidance behavior was never learned through early pain experiences. Chronic pressure ulcerations and osteomyelitis with bone destruction can occur (Figure 3). In some of these conditions the involvement of autonomic fibers causes limited heart rate variability, blood pressure fluctuations, an altered sweat secretion, and digestive problems. A few subtypes lead to neurogenic muscular atrophy and arthropathy. In addition, some patients have intellectual disability. The clinical features of the individual forms of HSAN are summarized in Table 1. Electrophysiological studies show axonal sensory neuropa- thy whereas motor nerve conduction studies and electromyography are often unremarkable or show only minor changes. A skin biopsy assessing nerve fiber density and sweat gland innervation can provide further diagnostic clues. Sural nerve biopsy can further classify the disorder, but is done less frequently in favor of molecular genetic diagnostics. There are some aspects that have to be considered in the molecular diagnostics such as a repeat structure in the PRDM12 gene (HSAN8) which can be expanded and is poorly covered in most exome-sequencing analyses or a mutational hotspot in a neuron-specific exon of the WNK1 gene (HSAN2) that is easily missed in routine diagnostics. Gene products involved in HSAN are diverse and affect sphingolipid metabolism, the shaping of lipid bilayers, and endoplasmic reticulum turnover via autophagy (ER-Phagy), neurotrophin signaling, or transcription [16]. Interestingly, distinct mutations in ELP1 lead to HSAN3, whereas other mutations have recently been described with medulloblastoma predisposition [17].

The therapy of HSAN is symptomatic. Conservative strategies for the treatment of ulcerations are important. Chronic ulceration and osteomyelitis can in some cases only be treated surgically, sometimes with the need for amputations, and is often associated with delayed wound healing, making both conservative and postoperative treatment long and difficult. In addition, patients do not have pain to moderate their behavior to allow both full wound healing and the avoidance of further injury - which manifests as Charcot's joints. Prophylactic measures such as regular examination for pressure sores and the smallest injuries should be carried out by patients or relatives to minimize the risk of infection. The treatment of autonomic dysfunction is the main focus of HSAN3 (gastrointestinal reflux, hypertension, bradyarrhythmias). Particularly in the case of HSAN4 with anhidrosis, overheating must be avoided and in children fever must be reduced quickly. Due to the strong tendency to self-mutilation, prophylactic tooth extraction of the milk teeth or a mouth guard should be considered. The wearing of gloves to avoid selfmutilation may also be indicated. Sufficient humidification of the eye and early treatment of corneal lesions is important for the preservation of vision. L-serine therapy represents the first targeted therapy option in SPTLC1associated HSAN1 [18].

\section{Pain gain}

So far, most of the monogenic pain disorders accompanied by severe, partly debilitating pain attacks or by progressive 


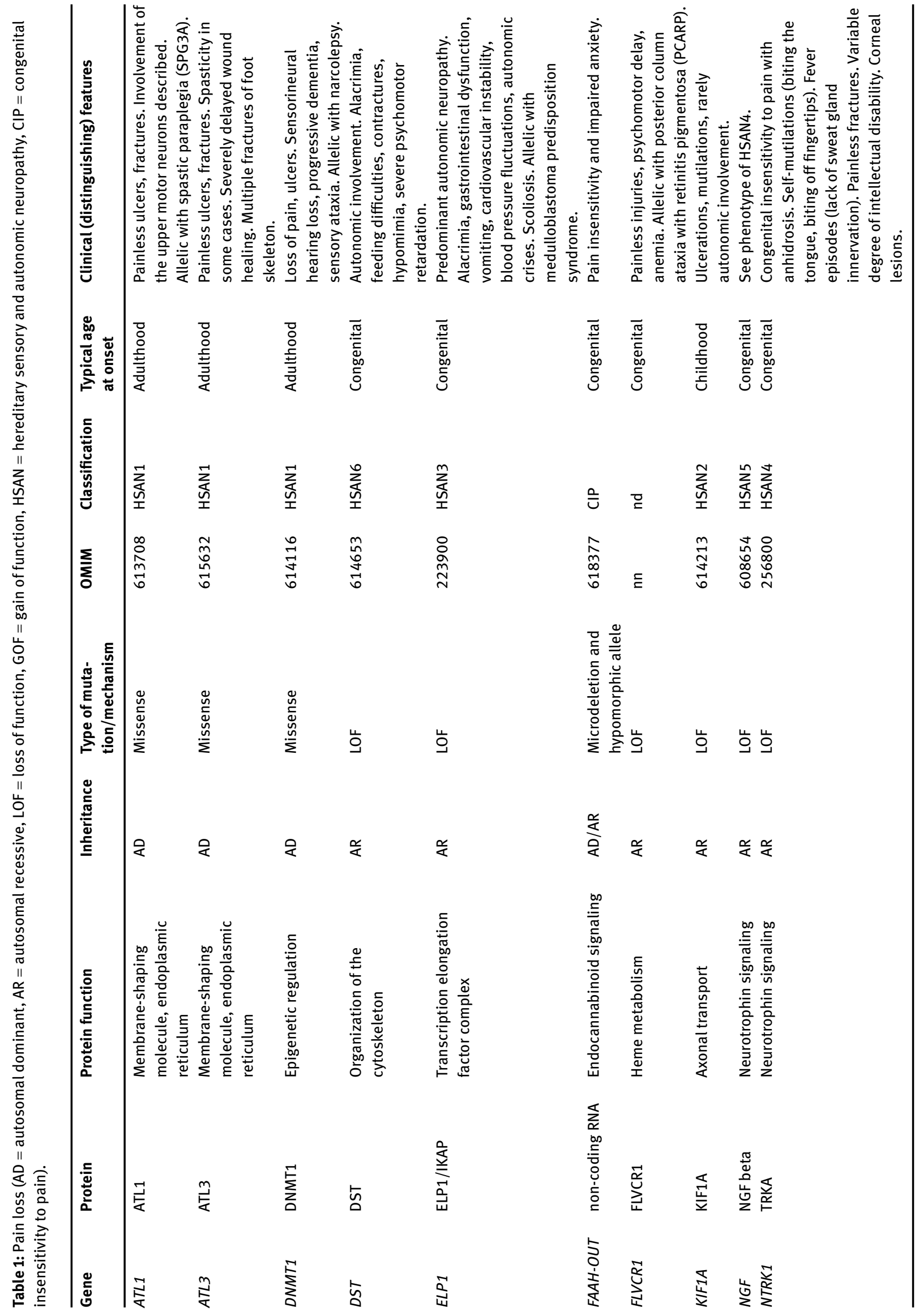




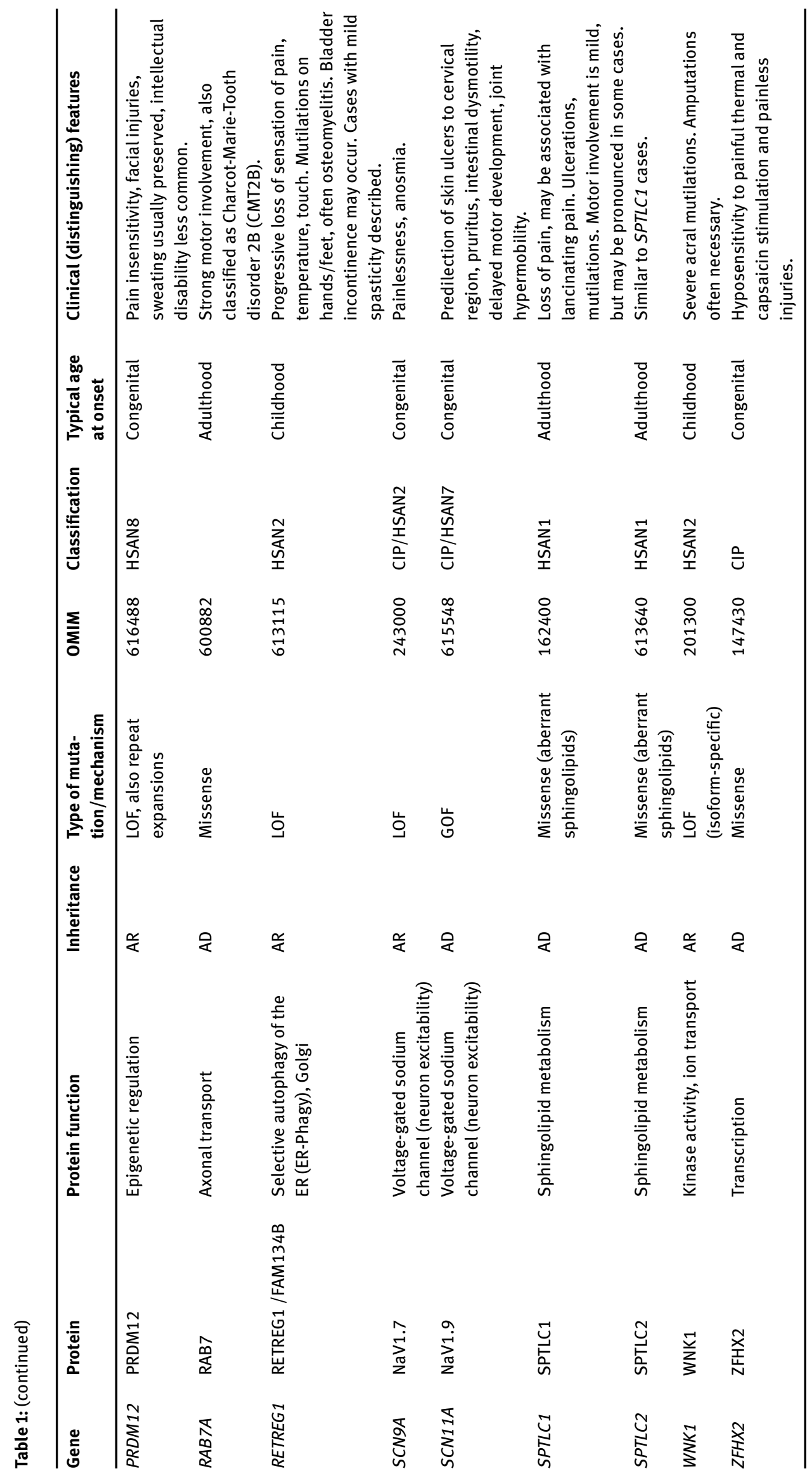


neurodegeneration of small sensory nerves are caused by mutations in ion channel genes. The genetics is complex since the same sodium channels implicated in pain loss can be involved in phenotypes associated with severe pain (Table 2). The pain can also only affect the extremities or inner organs.

Primary erythromelalgia (PE) is an autosomal dominant disorder typically presenting in early childhood and is caused by gain-of-function point mutations in $S C N 9 \mathrm{~A}$ $[19,20]$. Clinical symptoms include mild-to-severe burning pain, mostly in the hands and feet, increased skin temperature, edema, and erythema. Triggers such as warmth, exercise, prolonged standing, and alcohol are described. However, even with this typical medical history only $10 \%$ of PE patients are proven to have an SCN9A mutation [21]. Since analgesics, anticonvulsants, and antidepressants are not effective, patients often try to reduce pain by immersing the affected areas in ice-cold water. The sodium channel-blocking drug mexiletine is sometimes helpful, as are topical lidocaine plasters applied to the soles of the feet.

Paroxysmal extreme pain disorder (PEPD) is characterized by episodes of severe perineal and rectal, ocular, and mandibular pain. Age at onset is typically at birth or in infancy and triggers include defecation, eating, and sometimes strong emotion [22]. Autosomal-dominant gain-offunction mutations in $S C N 9 A$ that cause hyperexcitability of sensory neurons are responsible [23]. Carbamazepine is often effective at reducing the frequency and severity of painful attacks. Electrophysiological studies show that mutant $\mathrm{Na}_{\mathrm{V}} 1.7$ channels leading to PE tend to show lowered thresholds for the generation of action potentials and a higher frequency of repetitive firing, whereas mutants of $\mathrm{Na}_{V} 1.7$ causing PEPD rather make channel inactivation incomplete, resulting in a persistent current.

Familial episodic pain syndrome 1 (FEPS1) is characterized by debilitating upper body pain, yet only one large family has been described so far [24]. The autosomaldominant disease segregated with a gain-of-function missense mutation in a transmembrane segment of TRPA1, a chemosensitive transient receptor potential channel.

Familial episodic pain syndrome 2 (FEPS2) is caused by hyperexcitability-inducing missense mutations in $S C N 1 O A$, which encodes the $\mathrm{Na}_{\mathrm{V}} 1.8$ voltage-gated sodium channel. Patients have an adult-onset autosomaldominant paroxysmal pain disorder mainly affecting the legs and feet [25].

Familial episodic pain syndrome 3 (FEPS3) is another autosomal dominant episodic pain disorder caused by gain-of-function missense mutations in SCN11A $\left(\mathrm{Na}_{\mathrm{V}} 1.9\right)$ [11]. Pain is triggered by fatigue or exercise, shows partial relief by oral administration of anti-inflammatory analgesic drugs, and seems to improve with age in some cases. Of note, the distinction of FEPS2 and FEPS3 to small fiber neuropathies is not entirely clear.

Small fiber neuropathy (SFN) is typically an adultonset disorder characterized by burning pain which usually starts in the feet or hands, but can also affect other regions or lead to whole-body pain. It can be accompanied by abnormal skin sensations such as tingling or itchiness. In some individuals, the pain is more severe during times of rest or at night. Affected individuals have an increased sensitivity to pain in general (hyperalgesia) and experience pain from stimulation that typically does not cause pain (allodynia). Autonomic symptoms can include orthostatic dizziness, palpitations, dry eyes, and a dry mouth. Diabetes mellitus, increased consumption of alcohol, vitamin B12 deficiency, monoclonal gammopathy, vasculitis, or other systemic autoimmune disorders should be excluded before considering a genetic cause. Besides the medical history and physical examination, a punch biopsy of the skin is an important diagnostic criterion and typically shows a reduced intraepidermal nerve fiber density of small-diameter unmyelinated and thinly myelinated fibers. Large fiber function is preserved, which is reflected by inconspicuous nerve conduction studies. Quantitative sensory testing and tests for autonomic function can complete the diagnostic workup. Heterozygous gainof-function mutations in SCN9A, SCN1OA, and SCN11A have been reported in patients with SFN $[9,25,26]$. Numerous variants reported in association with SFN are relatively common in the general population and it is a matter of debate whether they rather represent benign variants, show reduced expressivity, or may act as risk factors with incomplete penetrance.

In the differential diagnosis particularly two monogenic pain disorders, i.e., familial amyloidotic polyneuropathy and Fabry disease, have to be considered since these painful sensory neuropathies are treatable.

Familial amyloidotic polyneuropathy (TTRrelated neuropathy): Autosomal-dominant amyloidotic polyneuropathy is a systemic disease with small and subsequently large fiber neuropathy and neuropathic pain. It shows variable penetrance and expressivity and an adult onset. Autonomic symptoms, muscle atrophy, cardiomyopathy, or renal insufficiency can complicate the disease. Early diagnosis and molecular confirmation of a TTR mutation is of importance, because tafamidis meglumine can be used as a TTR-stabilizing drug. Other therapeutic options include intravenous therapy with the small interfering RNA patisiran [27] or subcutaneous application of the antisense oligonucleotide inotersen [28]. 


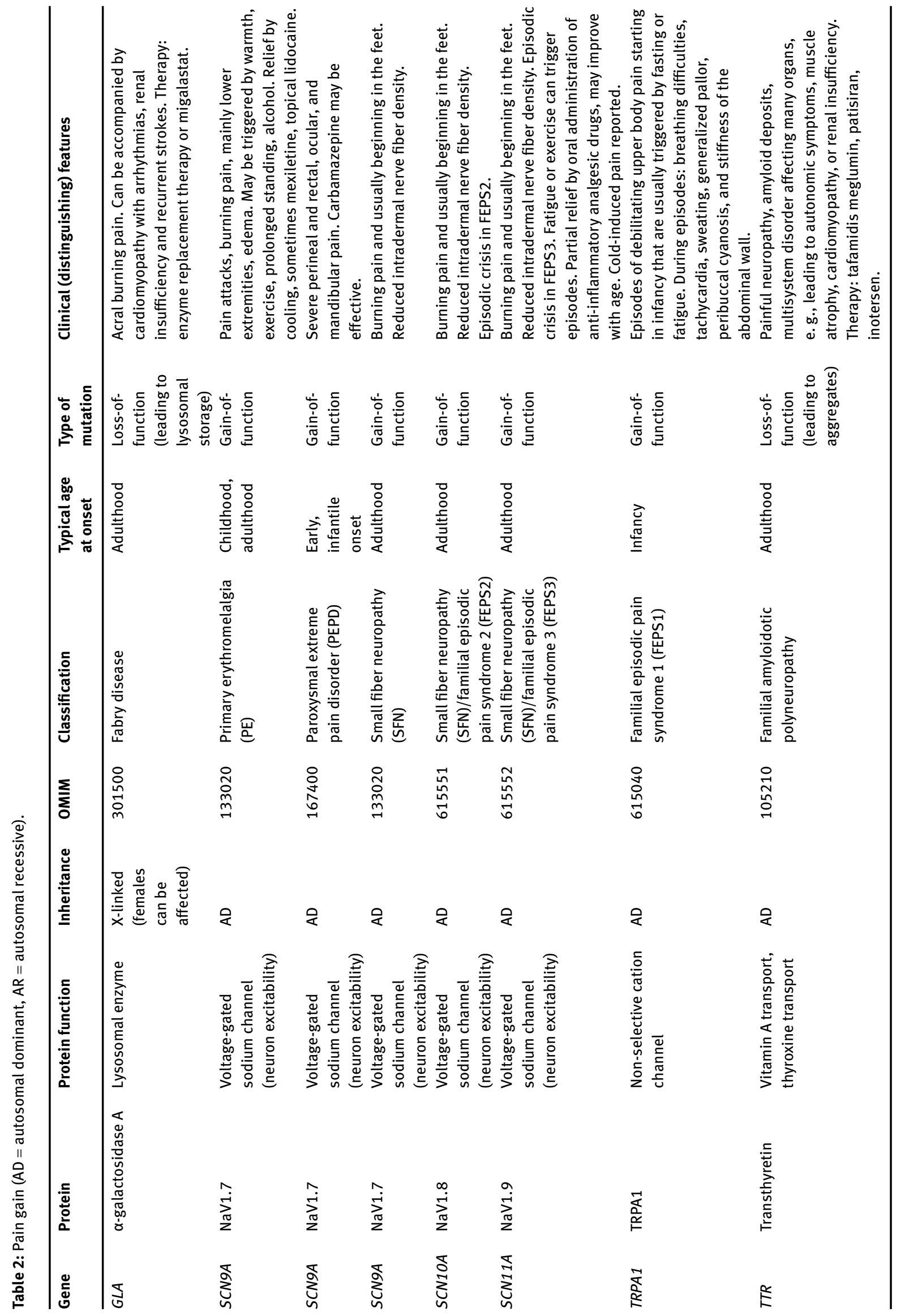


Table 3: Selected examples of analgesic drug candidates from monogenic pain loss disorders.

\begin{tabular}{llll}
\hline Target (gene/protein) & Drug candidate & Sponsor & Modality \\
\hline SCN9A/Nav1.7 & Vixotrigine & Biogen & Small molecule inhibitor \\
SCN9A/Nav1.7 & BIIB-095 & Biogen & Small molecule inhibitor \\
SCN9A/Nav1.7 & AM-6120, AM-8145, and AM-0422 & Amgen & Peptide derived from tarantula venom \\
SCN9A/Nav1.7 & VY-NAV-01 & Voyager Therapeutics & Gene therapy \\
NGF/NGF & Tanezumab & Pfizer, Eli Lilly & Monoclonal antibody \\
NGF/NGF & Fasinumab & Regeneron, Teva & Monoclonal antibody \\
NTRK1/TRKA & ASP7962 & Astellas Pharma & Small molecule inhibitor \\
NTRK1/TRKA & GZ389988A & Sanofi & Small molecule inhibitor \\
FAAH/FAAH & JNJ-42165279 & Johnson and Johnson & Small molecule inhibitor \\
\hline
\end{tabular}

Fabry disease: Fabry disease is an X-linked lysosomal storage disease which affects both males and females and is caused by mutations in the GLA gene, which codes for $\alpha$-galactosidase A. Episodic, mostly acral, burning pain is one of the first and main symptoms. Other symptoms include cardiomyopathy with arrhythmias, renal insufficiency, and recurrent strokes. Due to the manifold complications, early diagnosis with subsequent enzyme replacement therapy i. v. or Migalastat orally is crucial [29].

\section{Analgesic targets derived from monogenic pain disorders}

Mendelian pain insensitivity disorders are ideally suited to uncover pharmacological targets for potent analgesics and there is a huge unmet need for pain relief in the general population (Table 3). To block the voltage-gated sodium channel $\mathrm{Na}_{\mathrm{V}} 1.7$ is an obvious possibility since patients with $\mathrm{Na}_{\mathrm{V}} 1.7$ null mutations have complete analgesia but otherwise little additional symptoms. Significant reports have been made by the pharmaceutical industry to target $\mathrm{Na}_{V} 1.7$ [30], however, there are many pitfalls (subtype selectivity, degree of $\mathrm{Na}_{\mathrm{V}} 1.7$ inhibition) before such compounds may come into clinical use [31]. Links between $\mathrm{Na}_{\mathrm{V}} 1.7$ and opioid signaling may be used therapeutically as $\mathrm{Na}_{\mathrm{V}} 1.7$ blockers combined with low-dose opioids or enkephalinase inhibitors may produce profound analgesia [32]. In contrast, the human phenotype of $\mathrm{Na}_{\mathrm{V}} 1.9$ pain insensitivity illustrates that manipulating this channel systemically is likely to result in unwanted side effects. Moreover, the tiny window between channel hyperactivity that leads to analgesia and hyperactivity that leads to painful episodes could make it hard to find the right analgesic dose. Even if the target molecule leads to a morphological alteration of the nociceptors in the context of Mendelian pain loss (HSAN4, HSAN5), it may offer interesting approaches for pain therapy. Examples include clinical trials with nerve growth factor (NGF) or tropomyosin receptor kinase A (TRKA) antibodies [33].

\section{Conclusions}

Mendelian pain disorders reveal important aspects of human pain sensing. Patients with pain loss suffer from injuries and burns, and treatment is difficult. Several subtypes show additional clinical symptoms such as autonomic neuropathy, muscle weakness, or intellectual disability. Different molecular pathways are involved in pain insensitivity; however, voltage-gated sodium channels play a crucial role. The opposite phenotypic spectrum, i. e., Mendelian disorders with painful episodes or neuropathic pain, can be caused by different mutations in voltage-gated sodium channels. While treatment options are limited, it is important to diagnose treatable forms of pain-associated disorders such as familial amyloidotic polyneuropathy or Fabry disease. At the same time, Mendelian disorders of pain sensing identify critical pathways for analgesic targeting and their understanding has the potential to lead to novel analgesic classes with wide general applicability.

\section{References}

[1] McDermott LA et al. Defining the Functional Role of NaV1.7 in Human Nociception. Neuron. 2019;101(5):905-19. e8.

[2] Cox JJ et al. An SCN9A channelopathy causes congenital inability to experience pain. Nature. 2006;444(7121):894-8.

[3] Goldberg YP et al. Loss-of-function mutations in the Nav1.7 gene underlie congenital indifference to pain in multiple human populations. Clin Genet. 2007;71(4):311-9. 
[4] Weiss J et al. Loss-of-function mutations in sodium channel Nav1.7 cause anosmia. Nature. 2011;472(7342):186-90.

[5] Yuan J et al. Hereditary sensory and autonomic neuropathy type IID caused by an SCN9A mutation. Neurology. 2013;80(18):1641-9.

[6] Leipold $E$ et al. A de novo gain-of-function mutation in SCN11A causes loss of pain perception. Nat Genet. 2013;45(11):1399-404.

[7] King MK et al. Pain insensitivity: distal S6-segment mutations in NaV1.9 emerge as critical hotspot. Neurogenetics. 2017;18(3):179-81.

[8] Phatarakijnirund $V$ et al. Congenital insensitivity to pain: Fracturing without apparent skeletal pathobiology caused by an autosomal dominant, second mutation in SCN11A encoding voltage-gated sodium channel 1.9. Bone. 2016;84:289-98.

[9] Huang J et al. Gain-of-function mutations in sodium channel $\mathrm{Na}(v) 1.9$ in painful neuropathy. Brain. 2014;137(6):1627-42.

[10] Leipold E et al. Cold-aggravated pain in humans caused by a hyperactive NaV1.9 channel mutant. Nat Commun. 2015;6:10049.

[11] Zhang XY et al. Gain-of-function mutations in SCN11A cause familial episodic pain. Am J Hum Genet. 2013;93(5):957-66.

[12] Habib AM et al. A novel human pain insensitivity disorder caused by a point mutation in ZFHX2. Brain. 2018;141(2):365-76.

[13] Habib AM et al. Microdeletion in a FAAH pseudogene identified in a patient with high anandamide concentrations and pain insensitivity. Br J Anaesth. 2019;123(2):e249-53.

[14] Mikaeili $\mathrm{H}$ et al. CRISPR interference at the FAAH-OUT genomic region reduces $F A A H$ expression. bioRxiv. 2019. https://doi.org/10.1101/633396.

[15] Kurth I. Hereditary Sensory and Autonomic Neuropathy Type II. In: Adam MP et al., editors. GeneReviews((R)). Seattle (WA). 1993.

[16] Rotthier A et al. Mechanisms of disease in hereditary sensory and autonomic neuropathies. Nat Rev Neurol. 2012;8(2):73-85.

[17] Waszak SM et al. Germline Elongator mutations in Sonic Hedgehog medulloblastoma. Nature. 2020;580(7803):396-401.

[18] Fridman $\mathrm{V}$ et al. Randomized trial of l-serine in patients with hereditary sensory and autonomic neuropathy type 1 . Neurology. 2019;92(4):e359-70.

[19] Yang Y et al. Mutations in SCN9A, encoding a sodium channel alpha subunit, in patients with primary erythermalgia. J Med Genet. 2004;41(3):171-4.

[20] Bennett DL et al. The Role of Voltage-Gated Sodium Channels in Pain Signaling. Physiol Rev. 2019;99(2):1079-151.
[21] Goldberg YP et al. Human Mendelian pain disorders: a key to discovery and validation of novel analgesics. Clin Genet. 2012;82(4):367-73.

[22] Bennett DL, Woods CG. Painful and painless channelopathies. Lancet Neurol. 2014;13(6):587-99.

[23] Fertleman CR et al. SCN9A mutations in paroxysmal extreme pain disorder: allelic variants underlie distinct channel defects and phenotypes. Neuron. 2006;52(5):767-74.

[24] Kremeyer B et al. A gain-of-function mutation in TRPA1 causes familial episodic pain syndrome. Neuron. 2010;66(5):671-80.

[25] Faber CG et al. Gain-of-function Nav1.8 mutations in painful neuropathy. Proc Natl Acad Sci USA. 2012;109(47):19444-9.

[26] Faber CG et al. Gain of function NaV1.7 mutations in idiopathic small fiber neuropathy. Ann Neurol. 2012;71(1):26-39.

[27] Adams D et al. Patisiran, an RNAi Therapeutic, for Hereditary Transthyretin Amyloidosis. N Engl J Med. 2018;379(1):11-21.

[28] Benson MD et al. Inotersen Treatment for Patients with Hereditary Transthyretin Amyloidosis. N Engl J Med. 2018;379(1):22-31.

[29] Uceyler $\mathrm{N}$ et al. Characterization of pain in fabry disease. Clin J Pain. 2014;30(10):915-20.

[30] Sexton JE et al. The Genetics of Pain: Implications for Therapeutics. Annu Rev Pharmacol Toxicol. 2018;58:123-42.

[31] Kingwell K. Nav1.7 withholds its pain potential. Nat Rev Drug Discov. 2019. https://doi.org/10.1038/d41573-019-00065-0.

[32] Minett MS et al. Endogenous opioids contribute to insensitivity to pain in humans and mice lacking sodium channel Nav1.7. Nat Commun. 2015;6:8967.

[33] Miller RE, Block JA, Malfait AM. What is new in pain modification in osteoarthritis? Rheumatology (Oxford). 2018;57(suppl_4):iv99-iv107.

James J. Cox

Molecular Nociception Group, Wolfson Institute for Biomedical Research, University College London, London, UK j.j.cox@ucl.ac.uk

\section{Geoffrey Woods}

Cambridge Institute for Medical Research, The Clinical Medical School, University of Cambridge, Cambridge, UK

cw347@cam.ac.uk

\section{Ingo Kurth}

Institute of Human Genetics, Medical Faculty, Uniklinik RWTH Aachen, Pauwelsstr. 30, 52074 Aachen, Germany

ikurth@ukaachen.de 